\title{
Design of Indoor location System Based on Power Line Communication and RFID
}

\author{
Xiaojun Liu \\ The Chinese People's Armed Police Forces Academy \\ Langfang, Hebei ,China,065000 \\ wjxy_Ixj@163.com
}

Keywords: indoor location, RFID, power line communication, RS485

\begin{abstract}
The paper designs an indoor location system based on RFID and power line carrier technology, introduces the hardware structure and software system working principle. Use low-voltage power line inside the building to solve the field wiring and information transmission. Use RFID technology to get position and personnel information. The experiment shows that the system is stable, reliable, and easy to use.
\end{abstract}

\section{Introduction}

In recent years, an increasing number of complex fires happen in large buildings and sudden disaster. Facing up the flame, thermal radiation, smoke and toxic gases, darkness and complex structure, etc., fire officers and soldiers are prone to loss of orientation and even life danger, especially when affected under environmental and psychological factors. At the same time, it is difficult for commander in the rear fire rescue field to know of the actual situation of the scene fire and judge whether fire-fighters are in danger and their locations, so they can't give full and accurate command for fire fighting and rescue work. The increasingly complex structure is challenging firefighters in reconnaissance, fire fighting and rescue work, therefore, effective firefighter indoor location technology is necessary to make real-time locating and rescue fighters in the disaster according to the location information in order to reduce personnel casualties.

Typical indoor location technology includes radio frequency identification (RFID), ultra wideband (UWB), Bluetooth, wireless local area networks (WLAN), ZigBee, ultrasonic location, infrared location, near-field electromagnetic ranging (NFER) and so on, these solutions or systems transmit location information in the form of wire or wireless. But high temperature and smoke interference inside the buildings are much stronger, wireless transmission is often blocked by strong interference. Cable transmission mode needs more cost to lay wire respectively.

Due to the above problems, this paper puts forward indoor real-time locating scheme based on power line communication (PLC) technology and radio frequency identification (RFID) technology. The scheme resolves field wiring and information transmission by using low voltage power line in buildings as communication carrier, using RFID technology to get location and personnel information real time[1].

\section{System General Design}

The regional location system is composed with locator cards, monitoring nodes, master nodes and host computer, whose composition block diagram is as shown in Fig.1. The locator card, with personnel information, communications to monitoring node by wireless way. If fire fighter with RFID card enters the area where the antenna can be read in monitor node, locator card sends radio frequency carrier signal to monitor node. The monitor node antenna receives the radio-frequency signal, extracts and processes locator card information, finally transmits the locator card information and monitoring ID to the master node through power line. The master node transmits this information 
to the host computer through RS485 bus. So real-time information of indoor personnel can be recorded and the personnel distribution area also can be determined.

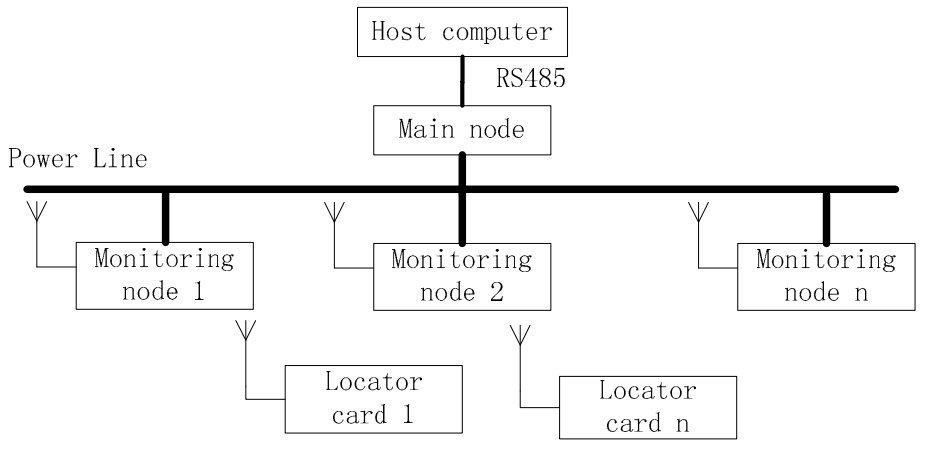

Fig 1. Block diagram of system construction

\section{Hardware Design of the System}

Monitoring node design. Monitoring node is composed by the radio frequency module, microprocessor and power carrier transceiver module.

RF module uses $2.4 \mathrm{GHz}$ ultra-low power consumption wireless transceiver chip nRF24L01 developed by the Nordic companies. With only a few peripheral components, the chip is very convenient because it has built-in address decoder, FILO stack, clock processing module, GFSK filter, and low noise amplifier. NRF24L01 has four work modes: transceiver model, configuration model, idle model and shutdown mode, which are controlled by three pins: PWR_UP, CE, TX_EN. Transceiver model has three ways: direct transceiver, ShockBrust TM and Enhanced ShockBurst TM[2]. To save energy, the system adopts Enhanced ShockBurstTM mode. NRF24L01 is in receiving mode after initialization. Once receiving new data, NRF24L01 determines whether the data are retransmitted signal in last time. The data are discarded if they are repeated data, or transmitted to microprocessor if they are confirmed to be effective new data[3]. The RF chip can check data, address code and remove the preamble code automatically. The microprocessor receives the RF chip data and transmits the data to power carrier interface. The connection between microprocessor and NRF24L01 is shown as Fig.2.

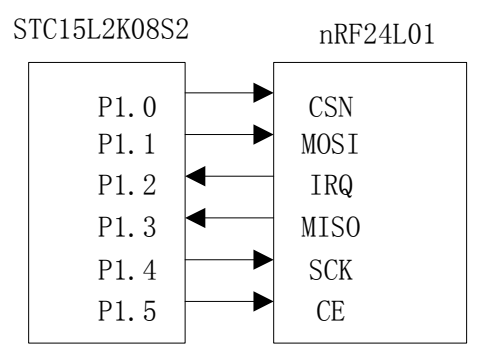

Fig. 2 connection between microprocessor and NRF24L01

The microprocessor uses single chip microcomputer STC15L2K08S2 produced by STC. Its instruction code is fully compatible with the traditional 8051, but the speed is faster with 8 to 12 times than the traditional 8051. STC15L2K08S2 has built-in 2k SRAM, 53k EEPROM, two groups of ultra-high speed asynchronous serial port and a set of synchronous serial port SPI. Because of only 3.3V power supply voltage, it wastes much less power than traditional 8051.

Power carrier module adopts power carrier chip ST7538 from the ST's. ST7538 integrates all the functionality of transmitting and receiving data, which can be easily connected to the microprocessor through serial communication. It also has automatic control and automatic voltage control, and can be connected with the power line through such minimal external devices as the coupling transformer. ST7538 also provides a watch dog, zero crossing detection, operational amplifier, clock output, timeout overflow output, $+5 \mathrm{v}$ power supply, etc., which greatly reduce the number of peripheral devices of ST7538 application circuit[4,5]. STC15L2K08S2 connects ST7538 with SPI interface. Data transmission direction is controlled by RxTx. When $\mathrm{RxTx}=0$, ST7538 is in a state of transmitting data. The data are sent into ST7538 from TXD, sampled at the rising edge of clock, and 
restituted in FSK modulator. Modulation signal is sent to the power line interface(PLI) circuit through D/A conversion, filter and ALC circuit, again through the differential amplifier by pin ATOP1 and ATOP2. When the $\mathrm{RxTx}=1, \mathrm{ST} 7538$ is in a state of receiving data. After signal is sent to ST7538 from the analog input, first through a band-pass filter to filter, then through AGC circuit of amplification which can adjust automatically according to the signal intensity of power line, and is sent into a narrow-band filter for filtering again, next are demodulated and filtered through the digital filter, and changed into serial digital signals sent to processor by RXD foot. When REG_DATA=1, read or write control register and set up the chip function parameters. The CLR_T outputs data synchronization clock signal, connects to the microcontroller external terminal. The microprocessor reads or writes ST7538 by interrupt. Each interrupt transmits or receives one data. If ST7538 detects the working frequency of the carrier, $\mathrm{CD} / \mathrm{PD}=0$; if no carrier, $\mathrm{CD} / \mathrm{PD}=1 . \mathrm{STC} 15 \mathrm{~L} 2 \mathrm{~K} 08 \mathrm{~S} 2$ and ST7538 connection is shown as Fig.3.

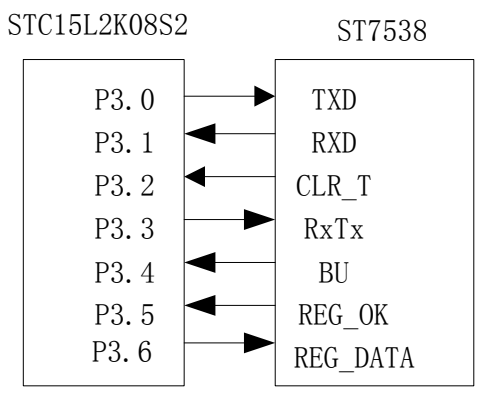

Fig.3 connection between STC15L2K08S2 and NRF24L01

Master node design.The master node is mainly composed of RS485 communication interface, microprocessor and power carrier communication interface. Among them, the power carrier communication interface is same to monitoring node, so they are no longer referred here. The microprocessor also adopts STC15L2K08S2.The RS485 communication interface adopts special communication chip SN75LBC184 which, as a RS485 interface chip from the American TI company, can accomplish the conversion between TTL and RS485. It works normally with a single power supply within $3-5.5 \mathrm{~V}$. Contrasted with other ordinary RS485 interface chips, SN75LBC184 has inside A and B pins connect with high-energy transient interference protection device have very good fighting effect on overvoltage transients caused by electrostatic discharge, and lightning. So it can be directly connected to communication lines without any protection components. In addition, its input impedance for the 2 times of RS485 standard impedance, so it can connect up to 64 communication bus transceivers. In order to improve the anti-interference performance of microprocessor STC15L2K08S2, high-speed optical coupler 6N137 is adopted for isolation between SN75LBC184 and STC15L2K08S2[6]. Master node's working principle is: The microprocessor STC15L2K08S2 detects whether monitoring nodes transmit carrier signal on power line. If signal presents, it will be received. The microprocessor monitor PC detects signal from RS485 bus at the same time, and transmits the relevant information with locator card and monitor node ID to the host computer when establishing a correspondence with PC.

\section{The Design Soft System}

Design of monitoring node program. Monitoring node implements two major functions: One is receiving personnel and location information as locator card is in the scope of its magnetic field. Location algorithm of the system is based on the strength of the received signal to measure distance, which uses radio signal strength with the propagation distance attenuation law to implement range measurement. The host computer software platform has registered and stored the location information of monitoring node ID. When the upload information includes locator card and monitoring node ID, which suggests that the locator card within the scope of effective monitoring, so as to determine the position of the locator card. The position is stored in the host computer for query and display. The locator card adopts the way of "label first speech". The situation that multiple 
locator cards send simultaneously information to the monitoring node can lead to the wireless channel information conflict, the monitoring node will not be able to read the data from locator cards. In fact, due to the transmission of only two bytes of the card number and one byte of voltage value, and the data transfer rate in the air by 2 MBPS, so the transmission time is too short. If many cards appear simultaneously in the same area, each card overlapping time difference is negligible. Based on this kind of situation, this system selects the Aloha as anti-collision algorithm method of cards. Once the locator card data conflict, the monitoring node will send instruction to terminate all cards send data information, and each card random delays sending again after a period of time. If many sets of different monitoring nodes send the same locator card information, which suggests that locator card is locating within the effective range with monitoring nodes, and the host computer makes comparisons according to the received signal strength and refresh the current effective position[7]. Another function of monitoring node is to detecting the carrier on power line; when power line is in idle state, monitoring node transmits the data information in the internal storage to the master node.

The Fig. 4 shows the flow chart of monitoring node. The program begins with system initialization which includes STC15L2K08S2, ST7538 and nRF24L01 initialization settings. Initialization of ST7538 mainly includes carrier frequency, baud rate and the detection sensitivity, etc.. Initialization of nRF24L01 includes model control, data channel, address width, automatic retransmission time, automatic reply function, working frequency, communication rate and transmission power settings, etc. Also, in order to communicate with locator card, there must be the same settings to locator card, with address width, working frequency and communication rate. After the initialization, set RF chip to receive state. Read the CD/PD state of ST7538 after receiving the locator card information. If the port data for 1 , that means the power line with other monitoring nodes are transmitting information to the master node, and the monitoring node stop temporarily data, and then repeat reading and judgment $\mathrm{CD} / \mathrm{PD}$ port data, until the port data is 0 , and then transmit locator card and monitoring node information to the master node.
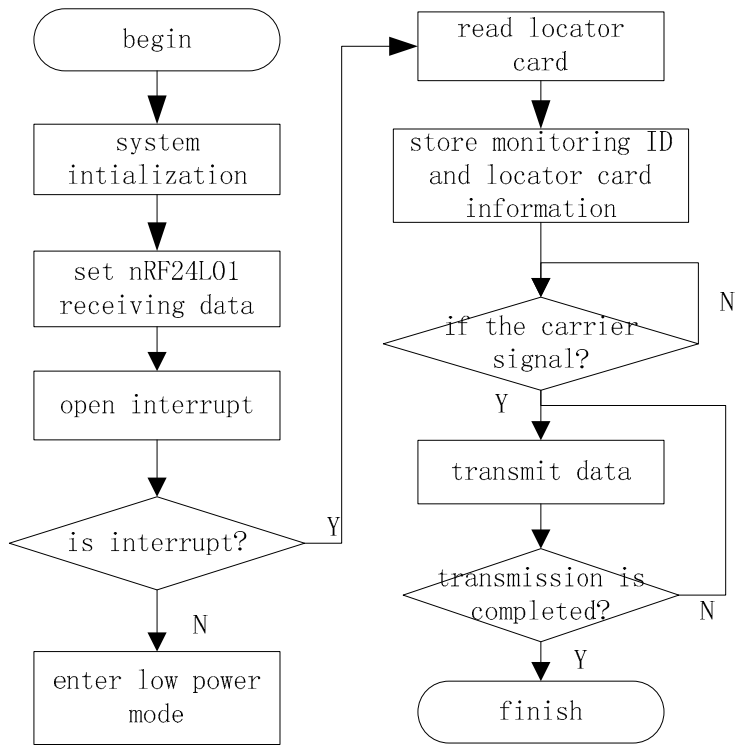

Fig.4 flow chart of monitoring node

Design of the master node program. The microprocessor communicates mainly with carrier chip ST7538 and PC. The communication with PC is through serial port. The communication with ST7538 includes ST7538 initialization settings, data transmitting and receiving from and to ST7538. The microprocessor transmits data to ST7538 by the way of interruption. After opening the interrupt, the module keeps waiting state. The microprocessor finishes receiving the data from power line, and then triggers the serial port to transmit interrupts. When PC transmits data to serial port, it will trigger receiving interrupt. In each interrupt service routine, the received data are stored in the data buffer[8]. All the received data are sent to a power line, and accordingly communication state changes. The flowchart of main node is shown as Fig.5. 


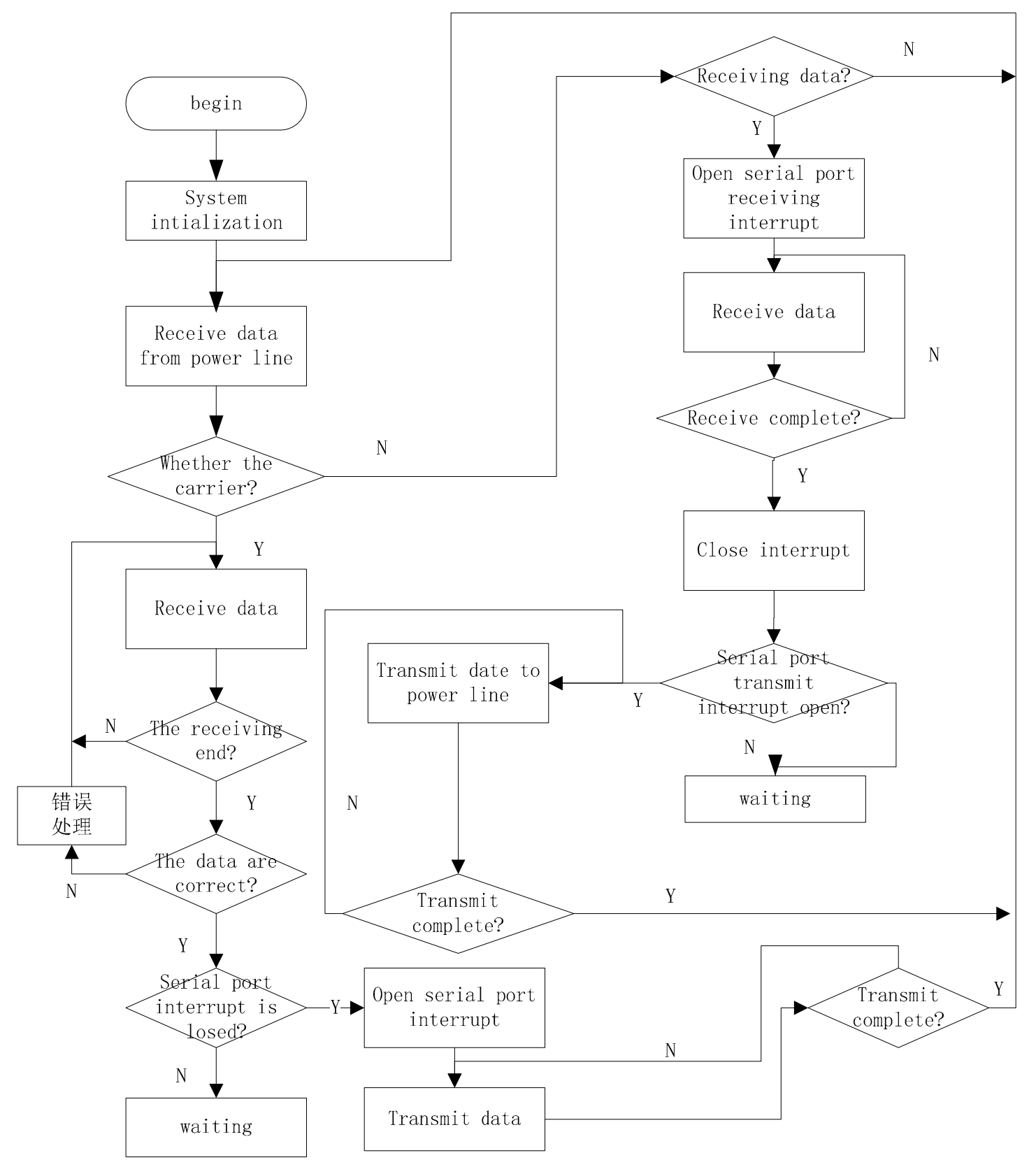

Fig.5 flow chart of master node

\section{Test results and analysis}

The RFID location function and power line carrier communication function were tested in the laboratory condition. The test results are shown in Table 1 and Table 2.

Table 1 the results of RFID location

\begin{tabular}{|c|c|c|c|c|}
\hline $\begin{array}{c}\text { Locator card } \\
\text { number }\end{array}$ & Distance $(\mathrm{m})$ & $\begin{array}{c}\text { Test } \\
\text { number }\end{array}$ & $\begin{array}{c}\text { Correct receiving } \\
\text { number }\end{array}$ & $\begin{array}{c}\text { Correct } \\
\text { recognition rate }\end{array}$ \\
\hline 1 & 5 & 150 & 150 & $100 \%$ \\
\hline 1 & 8 & 150 & 150 & $100 \%$ \\
\hline 1 & 10 & 150 & 149 & $99.3 \%$ \\
\hline 1 & 15 & 150 & 148 & $100 \%$ \\
\hline 3 & 8 & 150 & 150 & $100 \%$ \\
\hline 5 & 8 & 150 & 150 & \\
\hline
\end{tabular}


Table 2 the results of power line carrier communication

\begin{tabular}{|c|c|c|c|c|}
\hline $\begin{array}{c}\text { Test } \\
\text { distance(m) }\end{array}$ & $\begin{array}{c}\text { Transmit data } \\
\text { number }\end{array}$ & $\begin{array}{c}\text { Correct receiving } \\
\text { number }\end{array}$ & Correct rate & Load \\
\hline 10 & 3600 & 3600 & $100 \%$ & low \\
\hline 20 & 3600 & 3600 & $100 \%$ & low \\
\hline 30 & 3600 & 3589 & $99.7 \%$ & low \\
\hline 30 & 3600 & 3580 & $99.4 \%$ & heavy \\
\hline
\end{tabular}

Table 1 shows that one locator card at a distance less than $10 \mathrm{~m}$, the correct rate of monitoring node recognition reached $100 \%$. With the increasing of distance, the recognition correct rate declined slightly. For multiple locator cards, due to the adoption of the anti-collision algorithm, the recognition correct rate also reaches $100 \%$. These show the localization system is stable and reliable. Table 2 shows that the transmission error rate with low load is zero when the transmission distance is less than $20 \mathrm{~m}$. With the increase of the transmission distance, the error rate is increased. The loads impact the quality of the data transmission. The heavy load causes error rate high, because the attenuation of high frequency signal is large.

\section{Conclusion}

The indoor location and the transmission of location information are realized based on radio frequency and power line communication technology. The test results meet the design requirements. In addition, some external sensors can be connected to locator card to monitoring field environment, which provide decision-making reference for commander. The system test is completed in the laboratory environment. So further tests in the real simulation environment are needed in order to improve the performance of the system.

\section{References}

[1] ZHANG Yongping. Firefighter positioning system based on power line communication technique.Fire Science and Technology ,2015,Vol34:246-248.

[2]Nordic Semiconductor. nRF24L01 single chip $2.4 \mathrm{GHz}$ transceiver product specification [EB/OL]. (2007-XX-Xx) [2013-05-03].http : //www.nordicsemi.com/eng/Products/2.4GHz-RF/nRF24L01.

[3] Huang Xun,Ji Yancheng,Zhang Xiaoge. Design of E-reader Based on nRF24L01. Informatization on Research,2012.Vol38(4):58-60.

[4] STMicroelectronics.ST7538.2003.

[5] STMicroelectronics.ST7538 Application Note. 2003.

[6] Zhang Yizhe,Yang Xiaoqing, Ma Guoqing. Design of Security Alarm System Based on Power Line Carrier and RS485 Communication. Architecture Electric,2013,Vol32(10):50-55.

[7] Liu Zhiping. Personnel Attendance and Location Systemin Coal Mine Basedon RFID,2008, Harbin Engineering University.

[8] ZENG Suqiong, ZHANG Xuecheng. Design of Carrier Communication Module for Low Voltage Power Line Based on 89C2051 and ST7538. Low Voltage Appratus,2008,(11):33-36. 